\title{
Štefan Porubský
}

Natural exactly covering systems of congruences

Czechoslovak Mathematical Journal, Vol. 24 (1974), No. 4, 598-606

Persistent URL: http://dml.cz/dmlcz/101278

\section{Terms of use:}

(C) Institute of Mathematics AS CR, 1974

Institute of Mathematics of the Czech Academy of Sciences provides access to digitized documents strictly for personal use. Each copy of any part of this document must contain these Terms of use.

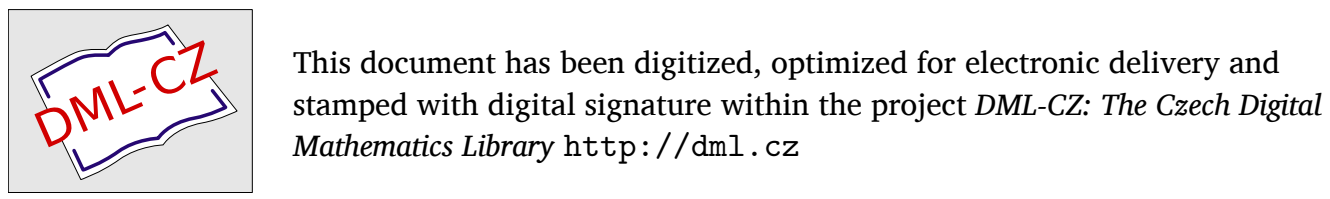




\title{
NATURAL EXACTLY COVERING SYSTEMS OF CONGRUENCES
}

\author{
S̆Tefan Porubský, Bratislava
}

(Received October 1, 1973)

\section{INTRODUCTION}

Throughout this paper an ordered pair of integers $(a, n)$ will denote the residual class $a(\bmod n)$. Moreover, we shall always suppose $0 \leqq a<n$ in this notation.

A system of residual classes

$$
\left(a_{i}, n_{i}\right), \quad i \in T
$$

will be called exactly covering if every integer belongs exactly to one of these classes.

Unless the contrary is stated we shall suppose $T=\{1,2, \ldots, k\}$.

The exactly covering systems (abbreviated ECS) are investigated in many papers, e.g. [1] $-[5]$. In [1] there is proved that every ECS contains at least two residual classes with respect to the greatest modulus. This result provided the original impetus to the study of ECS's (1) with the following property $\mathscr{P}(m)$ : The system $(1)$ contains exactly $m$ residual classes with respect to the greatest modulus while its all other residual classes have distinct moduli.

We can suppose $m>1$ in $\mathscr{P}(m)$ according to the mentioned result of [1].

The characterization of ECS's having property $\mathscr{P}(m)$ is not known in general, nevertheless the following particular cases have been solved: $m=2[3], m=3[4]$, $m=4,5$, and partly 7 [2]. Some of these results are collected in

Lemma 1. Let $m=2,3,5$ and let

in $\operatorname{ECS}(1)$. Then

$$
n_{1}<n_{2}<\ldots<n_{k-m}<n_{k-m+1}=\ldots=n_{k}
$$

$$
n_{i}=2^{i} \text { for } i=1,2, \ldots, k-m \text { and } n_{k-m+1}=\ldots=n_{k}=m .2^{k-m} .
$$

In this paper we shall define one subclass of ECS's - the natural exactly covering systems - and the equivalence between the mentioned problem and some diophantine equations in case of natural exactly covering systems will be shown. 


\section{DEFINITION OF NECS}

Let $0 \leqq a<b$. An $a b$-system $\Im_{a b}$ is a partially ordered system of residual classes constructed in the following manner:

1. The system $\{(a, b)\}$ is an $a b$-system.

2. Let $\mathfrak{\Im}_{a b}^{\prime}$ be an $a b$-system.

i) Let $\left(c_{1}, d\right), \ldots,\left(c_{t}, d\right)$ be distinct residual classes not belonging to $\mathfrak{\subseteq}_{a b}^{\prime}$ and $(e, f)$ a maximal element of $\mathfrak{\Xi}_{a b}^{\prime}$ with $\bigcup_{i=1}^{t}\left(c_{i}, d\right)=(e, f)$. Then $\mathfrak{\Xi}_{a b}=\mathfrak{\Xi}_{a b}^{\prime} \cup\left\{\left(c_{1}, d\right), \ldots\right.$ $\left.\ldots,\left(c_{t}, d\right)\right\}$ is again an $a b$-system.

ii) If there exist distinct maximal elements $\left(g_{1}, h\right), \ldots,\left(g_{r}, h\right)$ in $\mathfrak{\Xi}_{a b}^{\prime}$ with $\bigcup_{i=1}^{t}\left(g_{i}, h\right)=(c, n)$ for some $(c, n)$ not belonging to $\mathfrak{\Xi}_{a b}^{\prime}$ then $\mathfrak{\Xi}_{a b}=\mathfrak{\Xi}_{a b}^{\prime} \cup\{(c, n)\}$ is also an $a b$-system.

3. The ordering of $\mathfrak{\subseteq}_{a b}$ remains unchanged between any two elements of $\mathfrak{S}_{a b}^{\prime}$ and the newly added elements are incomparable in $(i)$ and in both cases thery are (or it is) greater than the corresponding maximal element (or elements) of $\mathfrak{\Xi}_{a b}^{\prime}$.

It is straightforward to verify that the system $\mathfrak{M}\left(\mathfrak{S}_{a b}\right)$ of all maximal elements of any $a b$-system $\subseteq_{a b}$ disjointly covers the residual class $(a, b)$. Thus the maximal elements of any 01-system form an ECS. The converse is also true.
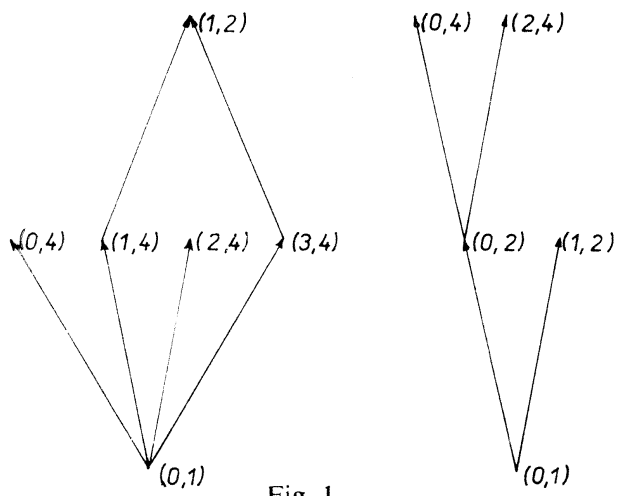

Fig. 1.

Lemma 2. Every ECS is formed by maximal elements of a suitable 01-system.

Proof. Let $n$ be the 1.c.m. $\left[n_{1}, \ldots, n_{k}\right]$ of moduli of ECS (1). Let us put

$$
\begin{aligned}
& \left.\mathfrak{\Im}_{01}^{(0)}=\{0,1)\right\} \cup\{(0, n), \ldots,(n-1, n)\}, \\
& \mathfrak{\Im}_{01}^{(i)}=\mathfrak{\Im}_{01}^{(i-1)} \cup\left\{\left(a_{i}, n_{i}\right)\right\}, \quad i=1,2, \ldots, k .
\end{aligned}
$$

Then $\mathfrak{M}\left(\mathfrak{\Im}_{01}^{(k)}\right)$ and $\operatorname{ECS}(1)$ coincide. 
It can happen that distinct 01-systems lead to identical ECS's. For instance, the 01 -systems given by their Hasse diagrams in Fig. 1 lead to the ECS $\{(1,2),(0,4)$, $(2,4)\}$.

An $a b$-system whose Hasse diagram is a (directed rooted) tree will be called natural. Thus a natural exactly covering system (abbreviated NECS) is a system of all maximal elements of a natural 01-system.

The introduced ordering of an $a b$-system $\mathfrak{S}_{a b}$ reduces to the converse ordering of that with respect to set inclusion in case of natural $\mathfrak{S}_{a b}$. Therefore in what follows the ordering of natural $a b$-systems will not be mentioned.

Every 01-system $\Im_{01}$ (not necessarily natural) can be transformed into an $a b$ system $\Im_{a b}\left(\Im_{01}\right)$ for every $0 \leqq a<b$ in the following manner:

$$
\mathfrak{\Im}_{a b}\left(\Im_{01}\right)=\left\{(a+c b, b d):(c, d) \in \mathfrak{\Xi}_{01}\right\}
$$

with the obvious ordering. This transformation corresponds to the revaluation of Hasse diagram of $\mathfrak{S}_{01}$.

Let $\Im_{01}$ be a natural 01-system. Then the outdegree of the root of its Hasse diagram is always greater than 1. For every vertex of a tree can be considered as a root of certain maximal subtree of the given one, the existence of suitable natural 01-systems $\mathfrak{S}_{01}^{(0)}, \ldots, \mathfrak{\Im}_{01}^{(n-1)}$ with

$$
\Im_{01}=\{(0,1)\} \cup \bigcup_{i=0}^{n-1} \Im_{i n}\left(\Im_{01}^{(i)}\right)
$$

can be easily verified, $n \geqq 2$ being the outdegree of the root of Hasse diagram of $\mathfrak{S}_{01}$.

It is easy to show that the g.c.d. of all moduli of a NECS equals at least the outdegree of roots of the corresponding trees and therefore it is greater than 1 . On this account the ECS

$$
\begin{gathered}
(0,6),(1,10),(2,15),(3,30),(4,30),(5,30),(7,30),(8,30),(9,30),(10,30), \\
(13,30),(14,30),(15,30),(16,30),(19,30),(20,30),(22,30),(23,30),(25,30), \\
(26,30),(27,30),(28,30),(29,30)
\end{gathered}
$$

cannot be natural. The ECS

$$
\begin{gathered}
(1,2),(0,12),(8,12),(2,20),(6,20),(10,20),(18,20), \\
(4,30),(14,60),(16,60),(28,60),(40,60),(52,60),(54,60)
\end{gathered}
$$

is an example of an ECS which is not natural though the g.c.d. of all its moduli is 2 . 
Considering a NECS $\mathfrak{M}\left(\Im_{01}\right)$ we shall always suppose $\mathfrak{\Xi}_{01}$ to be a natural 01system. If this 01 -system $\mathfrak{\Xi}_{01}$ is not essential for our consideration then we denote its corresponding NECS by $\mathfrak{M}$ only.

The first author who directed the attention to NECS's was $\breve{S}$. ZNÁM in his unpublished paper [5].

\section{MAIN THEOREMS}

Henceforward we shall investigate the NECS's only. In the following there turn out impracticable to hold at previous designation of moduli of $(1)$, i.e. $T=\{1, \ldots, k\}$, but we shall suppose $T$ to be a suitable finite set.

The small Roman characters (except possibly $x$ ) will denote natural numbers, the value 0 is admissible for $t$ and $i$ only; even $s$ with or without subscript will always denote natural number $>1$. The Greek character $\delta$ will denote 0 or 1 .

For our purpose, it shows very important to know in how many ways a natural number $m$ can be written in the form

$$
\begin{gathered}
m=k s-\sum_{i_{1}=1}^{k}\left\{\delta_{i_{1}} s_{i_{1}}+\sum_{i_{2}=1}^{r_{i_{1}}}\left[\delta_{i_{1} i_{2}} s_{i_{1} i_{2}}+\ldots+\right.\right. \\
\left.\left.+\sum_{i_{t}-1=1}^{r_{i_{1} \ldots i_{t}-2}}\left(\delta_{i_{1} \ldots i_{t-1}} s_{i_{1} \ldots i_{t-1}}+\sum_{i_{t}=1}^{r_{i_{1} \ldots i_{t}-1}} \delta_{i_{1} \ldots i_{t}} s_{i_{1} \ldots i_{t}}\right) \ldots\right]\right\}
\end{gathered}
$$

with the following properties:

1. if $t>0$ then $s_{i_{1}}$ is a proper divisor of $s$ for every $i_{1}$ while $s_{i_{1} \ldots i_{v}}$ is a proper divisor of $s_{i_{1} \ldots i_{v-1}}$ for every $i_{v}$ and $v=2, \ldots, t$;

2. the numbers $\delta_{i_{1}} s_{i_{1}}, \delta_{i_{1} i_{2}} s_{i_{1} i_{2}}, \ldots, \delta_{i_{1} \ldots i_{t}} s_{i_{1} \ldots i_{t}}$ which are different from zero are mutually distinct;

3. $r_{i_{1}}=\frac{s}{s_{i_{1}}}-\delta_{i_{1}}, \quad i_{1}=1, \ldots, k$,

$$
r_{i_{1} i_{2}}=\frac{s_{i_{1}}}{s_{i_{1} i_{2}}}-\delta_{i_{1} i_{2}}, \quad i_{2}=1, \ldots, r_{i_{1}},
$$

$$
r_{i_{1} \ldots i_{t-1}}=\frac{s_{i_{1} \ldots i_{t-2}}}{s_{i_{1} \ldots i_{t-1}}}-\delta_{i_{1} \ldots i_{t-1}}, \quad i_{t-1}=1, \ldots, r_{i_{1} \ldots i_{t-2}} .
$$

We say that the number $m$ is expressed in the form $\mathscr{F}_{t}(3)$ provided that $m$ is written in the form (3) and the above three conditions are satisfied. 
Theorem 1. Let $m$ be expressed in the form $\mathscr{F}_{t}(3)$ and $\delta=0$ or 1 . Then there exists a NECS with property $\mathscr{P}(m)$ whose all moduli are given by the relations

$$
\begin{aligned}
& n_{0}=\delta(k+\delta) \\
& n_{i_{1}}=\delta_{i_{1}}(k+\delta) \frac{s}{s_{i_{1}}}, \quad i_{1}=1, \ldots, k \\
& n_{i_{1} i_{2}}=\delta_{i_{1} i_{2}}(k+\delta) \frac{s}{s_{i_{1} i_{2}}}, \quad i_{2}=1, \ldots, r_{i_{1}}, \\
& \ldots \ldots \ldots \ldots . \ldots \ldots \ldots, \ldots \ldots, r_{i_{1} \ldots i_{t-1}}, \\
& n_{i_{1} \ldots i_{t}}=\delta_{i_{1} \ldots i_{t}}(k+\delta) \frac{s}{s_{i_{1} \ldots i_{t}}}, \quad i_{t}=1, \ldots \\
& n_{01}=n_{02}=\ldots=n_{0 m}=(k+\delta) s .
\end{aligned}
$$

Remark. The vanishing moduli appear in the statement of Theorem 1 only formally.

Proof. We give the proof by induction with respect to $t$. Let $t=0$. Then $m=k s$ with $s>1$. Let

$$
\mathfrak{S}_{01}^{(i)}=\{(0,1)\} \cup\{(0, s), \ldots,(s-1, s)\}, \quad i=0,1, \ldots, k-1,
$$

i.e., everyone of the corresponding NECS's $\mathfrak{M}\left(\mathfrak{S}_{01}^{(i)}\right)$ is the complete residue system modulo $s$. Besides, let

$$
\Im_{01}^{(k)}=\{(0,1)\}
$$

Then the set $\mathfrak{M}\left(\mathfrak{S}_{01}\right)$ of maximal elements of the natural 01 -system

$$
\Im_{01}=\{(0,1)\} \cup \bigcup_{i=0}^{k+\delta-1} \Im_{i(k+\delta)}\left(\Im_{01}^{(i)}\right)
$$

forms the required NECS.

Let our theorem hold for $t-1$, we prove it for $t$. After a simple modification we get

$$
m=\sum_{i_{1}=1}^{k}\left\{\left(\frac{s}{s_{i_{1}}}-\delta_{i_{1}}\right) s_{i_{1}}-\sum_{i_{2}=1}^{r_{i_{1}}}\left[\delta_{i_{1} i_{2}} s_{i_{1} i_{2}}+\ldots+\sum_{i_{t}=1}^{r_{i_{1}} \ldots i_{t-1}} \delta_{i_{1} \ldots i_{t}} s_{i_{1} \ldots i_{t}}\right]\right\}=\sum_{i_{1}=1}^{k} m_{i_{1}},
$$

where $m_{i_{1}}$ denotes the expression in braces. It is evident that $m_{i_{1}}$ is expressed in the form $\mathscr{F}_{t-1}(3)$ for every $i_{1}=1, \ldots, k$. Then there exists a NECS $\mathfrak{M}\left(\mathfrak{S}_{01}^{\left(i_{1}-1\right)}\right)$ with 
property $\mathscr{P}\left(m_{i_{1}}\right)$ owing to induction hypothesis and the moduli of these systems are given by

$$
\begin{aligned}
& n_{i_{1}}=\delta^{\left(i_{1}\right)}\left(\frac{s}{s_{i_{1}}}-\delta_{i_{1}}+\delta^{\left(i_{1}\right)}\right), \\
& n_{i_{1} i_{2}}=\delta_{i_{1} i_{2}}\left(\frac{s}{s_{i_{1}}}-\delta_{i_{1}}+\delta^{\left(i_{1}\right)}\right) \frac{s_{i_{1}}}{s_{i_{1} i_{2}}}, i_{2}=1, \ldots, r_{i_{1}}, \\
& \ldots \ldots \ldots \ldots \\
& n_{i_{1} \ldots i_{t}}=\delta_{i_{1} \ldots i_{t}}\left(\frac{s}{s_{i_{1}}}-\delta_{i_{1}}+\delta^{\left(i_{1}\right)}\right) \frac{s_{i_{1}}}{s_{i_{1} \ldots i_{t}}}, i_{t}=1, \ldots, r_{i_{1} \ldots i_{t-1}}, \\
& n_{01}=\ldots=n_{0 m_{i_{1}}}=\left(\frac{s}{s_{i_{1}}}-\delta_{i_{1}}+\delta^{\left(i_{1}\right)}\right) s_{i_{1}}
\end{aligned}
$$

for every $i_{1}=1, \ldots, k$. Let us put $\delta^{\left(i_{1}\right)}=\delta_{i_{1}}$ in each of these relations. Then the greatest moduli of all the systems $\mathfrak{M}\left(\mathfrak{S}_{01}^{(0)}\right), \mathfrak{M}\left(\mathfrak{S}_{01}^{(1)}\right), \ldots, \mathfrak{M}\left(\mathfrak{\Im}_{01}^{(k-1)}\right)$ are mutually equal. Let

$$
\Im_{01}=\{(0,1)\} \cup \bigcup_{i=0}^{k+\delta-1} \Im_{i(k+\delta)}\left(\Im_{01}^{(i)}\right)
$$

with $\mathfrak{S}_{01}^{(k)}=\{(0,1)\}$. Then $\mathfrak{M}\left(\mathfrak{S}_{01}\right)$ is the system satisfying the statement of our theorem and the proof is complete.

The next theorem shows that Theorem 1 can be inverted. However, we shall still need one notion to prove this fact. Let $\Xi_{01}$ be a natural 01-system. Then we can define the distance $\varrho\left(x_{0}, x\right)$ between an arbitrary element $x \in \Xi_{01}$ and the root $x_{0}=(0,1)$ as the cardinality of the set $\left\{x_{1} \in \mathfrak{S}_{01}: x_{0}<x_{1} \leqq x\right\}$, i.e., $\varrho\left(x_{0}, x\right)$ denotes the length of arc from $x_{0}$ to $x$ in Hasse diagram of $\mathfrak{\Xi}_{01}$.

Let $\mathfrak{M}$ be a NECS. Let us put

$$
h(\mathfrak{M})=\min \left\{\max \left\{\varrho\left(x_{0}, x\right): x \in \mathfrak{S}_{01}, x_{0}=(0,1)\right\}\right\},
$$

where the minimum is taken over all natural systems $\mathfrak{S}_{01}$ with $\mathfrak{M}=\mathfrak{M}\left(\mathfrak{G}_{01}\right)$. A positive integer $h(\mathfrak{M})$ determined in this way will be called the height of the NECS $\mathfrak{M}$. For instance, if $h(\mathfrak{M})=1$ then $\mathfrak{M}$ is a complete residue system.

Theorem 2. Let $\mathfrak{M}$ be a NECS having property $\mathscr{P}(m)$. Then $m$ is expressible in the form $\mathscr{F}_{t}(3)$ in such a way that all the moduli of $\mathfrak{M}$ are given by the corresponding relations (4) with a suitable $\delta$.

Proof. As to $\delta$ we prove, if $h(\mathfrak{M})>1$ and $k=1$ in (3) then $\delta=1$ in (4) and $\delta=0$ or 1 otherwise. 
We shall proceed by induction with respect to the height $h(\mathfrak{M})$ of the given NECS $\mathfrak{M}$. Let $h(\mathfrak{M})=1$. Then $\mathfrak{M}$ is a complete residue system, say modulo $s(s>1)$. In this case we get the statement of Theorem for $t=0, m=1 s, \delta=0$.

Let $h(\mathfrak{M})=2$ and let $\mathfrak{M}=\mathfrak{M}\left(\mathfrak{S}_{01}\right)$ with such 01 -system $\mathfrak{S}_{01}$ for which

$$
\max \left\{\varrho\left(x_{0} . x\right): x \in \subseteq_{01}, x_{0}=(0,1)\right\}=2 .
$$

We can easily verify that this $\mathfrak{S}_{01}$ has the form from Fig. 2 and therefore

$$
\begin{aligned}
& \mathfrak{\Im}_{01}^{(i)}=\{(0,1)\} \cup\{(0, b), \ldots,(b-1, b)\}, \quad i=0,1, \ldots, n-2, \\
& \mathfrak{\Im}_{01}^{(n-1)}=\{(0,1)\}
\end{aligned}
$$

for this $\mathfrak{\Im}_{01}$ in (2) with some $b>1$. Hence it is sufficient to put $\delta=1, s=b$, $k=n-1, t=0$ to obtain the statement of theorem.

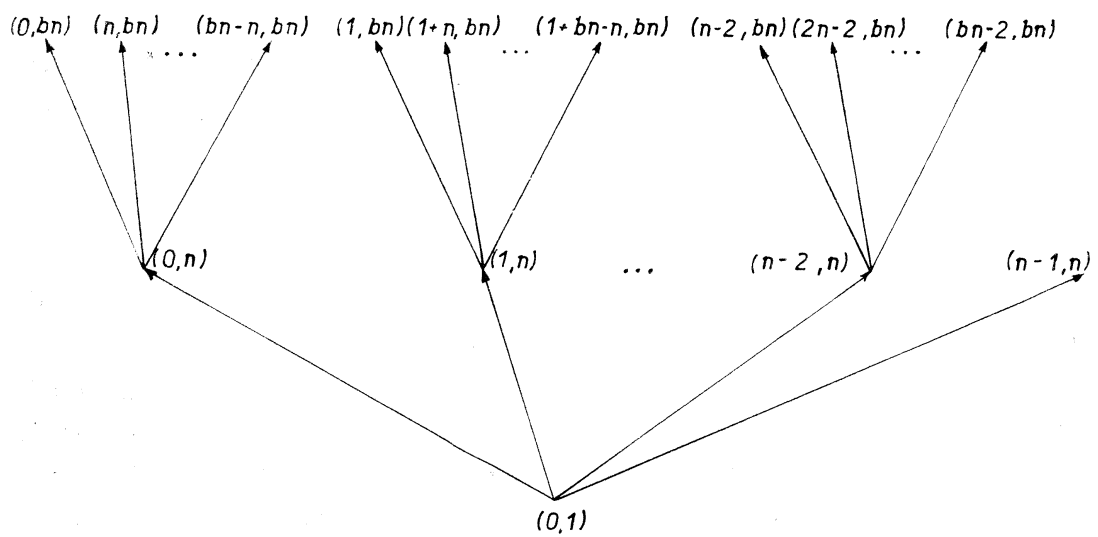

Fig. 2.

Let $\mathfrak{M}=\mathfrak{M}\left(\mathfrak{\Im}_{01}\right)$ be a NECS with $h(\mathfrak{M}) \geqq 3$ and with an $\mathfrak{\Im}_{01}$ for which the height of $\mathfrak{M}$ is attained. Let $\mathfrak{\Xi}_{01}^{(0)}, \ldots, \mathfrak{\Im}_{01}^{(n-1)}$ be 01 -systems from $(2)$ for this 01 -system $\mathfrak{S}_{01}$. Then at most one of them can be of the form $\{(0,1)\}$. Let $\mathfrak{S}_{01}^{(0)}, \ldots, \mathfrak{S}_{01}^{(n-1-\delta)}$ be all of them which are not of this form with $\delta=0$ or 1 . Let $k=n-\delta$. If $k=1$ then $\delta=1$ because $n \geqq 2$ in (2). Each of NECS's $\mathfrak{M}_{1}=\mathfrak{M}\left(\mathfrak{S}_{01}^{(0)}\right), \ldots, \mathfrak{M}_{k}=\mathfrak{M}\left(\mathfrak{S}_{01}^{(n-1-\delta)}\right)$ is of the same character as $\mathfrak{M}$, i.e, $\mathfrak{M}_{i_{1}}$ has property $\mathscr{P}\left(m_{i_{1}}\right)$ with some $m_{i_{1}}\left(i_{1}=\right.$ $=1, \ldots, k)$ for which $m=\sum m_{i_{1}}$. The greatest moduli of all the systems $\mathfrak{M}_{i_{1}}$ are mutually equal and $h\left(\mathfrak{M}_{i_{1}}\right)<h(\mathfrak{M})$ for every $i_{1}=1, \ldots, k$. Therefore the number $m_{i_{1}}$ is expressible in the form

$$
\left.m_{i_{1}}=r_{i_{1}} s_{i_{1}}-\sum_{i_{2}=1}^{r_{i_{1}}}\left[\delta_{i_{1} i_{2}} s_{i_{1} i_{2}}+\ldots+\sum_{i_{t}=1}^{r_{i_{1} \ldots i_{t}-1}} \delta_{i_{1} \ldots i_{t}} s_{i_{1} \ldots i_{t}}\right) \ldots\right]
$$


so that all moduli of NECS $\mathfrak{M}_{i_{1}}$ are given by the relations

$$
\begin{aligned}
& n_{i_{1}}=\delta_{i_{1}}\left(r_{i_{1}}+\delta_{i_{1}}\right), \\
& n_{i_{1} i_{2}}=\delta_{i_{1} i_{2}}\left(r_{i_{1}}+\delta_{i_{1}}\right) \frac{s_{i_{1}}}{s_{i_{1} i_{2}}}, \quad i_{2}=1, \ldots, r_{i_{1}}, \\
& \ldots \ldots \ldots \ldots \ldots \ldots \ldots \ldots \ldots \ldots \ldots \ldots \ldots \ldots \ldots \ldots \ldots r_{i_{1} \ldots i_{t-1}}, \\
& n_{i_{1} \ldots i_{t}}=\delta_{i_{1} \ldots i_{t}}\left(r_{i_{1}}+\delta_{i_{1}}\right) \frac{s_{i_{1}}}{s_{i_{1} \ldots i_{t}}}, \quad i_{t}=1, \ldots=n_{0 m_{i_{1}}}=\left(r_{i_{1}}+\delta_{i_{1}}\right) s_{i_{1}}
\end{aligned}
$$

with a suitable $\delta_{i_{1}}$ for every $i_{1}=1, \ldots, k$. Since the greatest moduli of these all NECS's are equal, it is

$$
\left(r_{1}+\delta_{1}\right) s_{1}=\ldots=\left(r_{k}+\delta_{k}\right) s_{k} \quad(=s) .
$$

We show now that $s_{1}, \ldots, s_{k}$ are proper divisors of $s$.

If either $r_{i_{1}}>1$ or $h\left(\mathfrak{M}_{i_{1}}\right)>1$ then always $r_{i_{1}}+\delta_{i_{1}}>1$ owing to the induction assumption and therefore $s_{i_{1}}$ is a proper divisor of $s$.

Let $h\left(\mathfrak{M}_{i_{1}}\right)=1$ and $r_{i_{1}}=1$ for some $i_{1}=1, \ldots, k$. Since $h\left(\mathfrak{M}_{i_{1}}\right)=1$, the NECS $\mathfrak{M}_{i_{1}}$ is the complete residue system and therefore $t=0$ in (6) owing to the first step of this proof. If $s_{i_{1}}$ is a composite number then (6) can be rewritten so that $r_{i_{1}}>1$ and $s_{i_{1}}>1$ without changing (7) because $\delta_{i_{1}}=0$. Hence $r_{i_{1}}+\delta_{i_{1}}>1$. Let $s_{i_{1}}$ be a prime number. Then $\mathfrak{M}_{i_{1}}$ is the complete residue system modulo prime number $s_{i_{1}}$. On the other hand, $\mathfrak{M}$ has property $\mathscr{P}(m)$, i.e., $\mathfrak{M}$ contains exactly $m$ residual classes with respect to the same modulus while the moduli of the other classes are distinct. Then the set of residual classes with respect to the greatest modulus of all systems $\mathfrak{M}_{i_{1}}\left(i_{1}=1, \ldots, k\right)$ must be modulo this prime $s_{i_{1}}$ and therefore each of $\mathfrak{M}_{i_{1}}$ 's is the complete residue system modulo $s_{i_{1}}$, because all the moduli of any NECS (even of any ECS) are equal if its greatest modulus is a prime. But then $h(\mathfrak{M}) \leqq$ $\leqq 2$ which is a contradiction.

Hence $s_{1}, \ldots, s_{k}$ are really proper divisors of $s$. Now we obtain the statement of Theorem considering (2) and

$$
r_{i_{1}} s_{i_{1}}=s-\delta_{i_{1}} s_{i_{1}}, \quad i_{1}=1, \ldots, k
$$

\section{NOTES}

Every positive integer $m$ is expressible as

$$
m=1 \cdot\left(m 2^{t}\right)-\left[1 \cdot\left(m 2^{t-1}\right)+\ldots+1 \cdot m\right],
$$

consequently every $m>1$ can be expressed in the form $\mathscr{F}_{t}(3)$ with any $t \geqq 0$. But this representation is unique for some $m$.

Theorem 3. For every $t \geqq 0$ the integer 2 can be uniquely expressed in the form $\mathscr{F}_{t}(3)$. Namely

$$
k=1, \quad s=2 \text { for } t=0
$$


and

$$
k=1, \quad s=2^{t+1}, \quad \underbrace{\delta_{1 \ldots 1}}_{i \text {-times }}=1, \quad \underbrace{s_{1 \ldots 1}}_{i \text {-times }}=2^{t+1-i} \text { for } i=1, \ldots, t \text { if } t \geqq 1 .
$$

Proof. We prove the uniqueness by induction with respect to $t$. It is straightforward to show our theorem in case $t=0$. Let the number 2 be expressed in the form $\mathscr{F}_{t}(3)$. Using (5) we get

$$
2=m_{1}+m_{2}+\ldots+m_{k}
$$

with all $m_{i}$ 's expressible in the form $\mathscr{F}_{t-1}(3)$. On the other hand we can easily prove that $m>1$ if it is expressible in the form $\mathscr{F}_{t}(3)$, and therefore $k=1$. Rewritting the equation (3) in the form

$\left.2=\left(s / s_{1}-\delta_{1}\right) s_{1}-\sum_{i_{2}=1}^{r_{1}}\left[\delta_{1, i_{2}} s_{1, i_{2}}+\ldots+\sum_{i_{\mathrm{t}}=1}^{r_{1, i_{2} \ldots i_{t}-1}} \delta_{1, i_{2} \ldots i_{t}} s_{1, i_{2} \ldots i_{t}}\right) \ldots\right]$

we obtain

$s / s_{1}-\delta_{1}=1, \quad s_{1}=2^{t}, \quad \delta_{i \text {-times }}^{\delta_{1 \ldots 1}}=1, \quad s_{\underbrace{1 \ldots 1}_{i-\text { times }}}=2^{t-(i-1)}$ for $i=2,3, \ldots, t$

owing to the induction hypothesis. Since $s_{1}$ is a proper divisor of $s, \delta_{1}=1$ and therefore $s / s_{1}=2, s=2^{t+1}$ and the proof is complete.

Corollary. Let (1) be a NECS containing exactly one couple of residual classes with respect to the greatest modulus while the moduli of its other residual classes are distinct. Then (changing the indices if necessary)

$$
n_{i}=2^{i} \text { for } i=1, \ldots, t-2 \text { and } n_{t-1}=n_{t}=2^{t-1} .
$$

STEIN has proved in [3] that the ECS's satisfying the assumptions of Corollary have just this form and therefore the NECS's exhaust all ECS's in this case. Moreover, the ECS's included in the results of [2] and [4] can be shown to be natural, too. Nevertheless, not every ECS with property $\mathscr{P}(m)$ is natural as is shown by our example of ECS which is not natural.

\section{References}

[1] Erdös, P.: Egy kongruenciarendszerekről szóló problémáról. Mat. Lapok 3, 122-128 (1952).

[2] Porubský, $\check{S}$.: Generalization of some results for exactly covering systems. Mat. časopis 22, 208-214 (1972).

[3] Stein, S. K.: Unions of arithmetic sequences. Math. Ann. 134, 289-294 (1954).

[4] Znám, S.: On exactly covering systems of arithmetic sequences. Math. Ann. 180, 227-232 (1969).

[5] Znám, Š.: On relations between exactly covering systems and rooted trees. Unpublished.

Author's address: 88625 Bratislava, ul. Obrancov mieru 41, ČSSR (Matematický ústav SAV). 